\title{
First-line chemotherapy in patients with metastatic gastroenteropancreatic neuroendocrine carcinoma
}

This article was published in the following Dove Press journal:

OncoTargets and Therapy

3 December 2015

Number of times this article has been viewed

\author{
Alberto Bongiovanni' \\ Nada Riva' \\ Marianna Ricci' \\ Chiara Liverani' \\ Federico La Manna' \\ Alessandro De Vita' \\ Flavia Foca ${ }^{2}$ \\ Laura Mercatali' \\ Stefano Severi ${ }^{3}$ \\ Dino Amadori ${ }^{4}$ \\ Toni Ibrahim' \\ 'Osteoncology and Rare Tumors \\ Center, ${ }^{2}$ Unit of Biostatistics and \\ Clinical Trials, ${ }^{3}$ Nuclear Medicine Unit, \\ ${ }^{4}$ Department of Medical Oncology, \\ Istituto Scientifico Romagnolo per lo \\ Studio e la Cura dei Tumori (IRST) \\ IRCCS, Meldola, FC, Italy
}

\begin{abstract}
Background and aim: To investigate the efficacy of platinum-based chemotherapy in patients with metastatic gastroenteropancreatic neuroendocrine carcinoma (mGEP-NEC) and define predictive and prognostic factors.

Methods: Twenty mGEP-NEC patients were treated with cisplatin or carboplatin/etoposide between April 2010 and October 2014. Both large-cell and small-cell histologies were included. Cisplatin $25 \mathrm{mg} / \mathrm{m}^{2}$ was administered on days 1-3 followed by etoposide $100 \mathrm{mg} / \mathrm{m}^{2}$ on days $1-3$ every 21 days. Carboplatin $300 \mathrm{mg} / \mathrm{m}^{2}$ was administered on day 1 followed by etoposide $100 \mathrm{mg} / \mathrm{m}^{2}$ on days $1-3$.
\end{abstract}

Results: Of the 19 evaluable patients, 13 obtained a partial response, four showed stable disease, and two progressed. Median overall survival (mOS) was 13.5 months and median progressionfree survival (mPFS) was 10.9 months. Gallium-68 positron emission tomography/computerizsed tomography-positive patients had a higher, albeit not significantly, OS than those with negative results (75\% vs $34.3 \%$ at 18 months; $P=0.06)$. mPFS was 19.3 and 6.3 months $(P<0.01)$ in mGEP-NEC patients with Ki67 $\leq 55 \%$ or $>55 \%$, respectively. mOS was 8.1 months in the latter group but was not reached in the Ki67 $\leq 55 \%$ group $(P$-value $=0.039)$. Patients with a lower body mass index (BMI) had a better prognosis in terms of both OS and PFS. Patients with BMI $\geq 25$ had a mOS of 11.7 months $(P=0.0293)$ and a mPFS of 6.2 months $(P=0.0057)$.

Conclusion: Platinum-based chemotherapy showed good efficacy in mGEP-NEC patients. Those with Ki67 $\leq 55 \%$, positive Gallium-68 positron emission tomography/computerized tomography and BMI $<25$ had a better prognosis.

Keywords: chemotherapy, gastroenteropancreatic neuroendocrine carcinoma, platinum-based chemotherapy

\section{Introduction}

Neuroendocrine neoplasia (NEN) of the gastroenteropancreatic (GEP) tract is a heterogeneous group of malignancies. ${ }^{1}$ The 2010 World Health Organization (WHO) classification divides these neoplasms into three groups according to their $\mathrm{Ki} 67 \%$ value: well-differentiated and moderately differentiated neuroendocrine tumors (NETs; G1 and G2 NETs, respectively) for Ki67 $\leq 20 \%$ and neuroendocrine carcinomas (NECs) for Ki67 $>20 \%{ }^{2}$ The latter group includes small- and large-cell NECs and mixed components of adenocarcinoma or other non-neuroendocrine elements may also be associated with either of these NEC subtypes. ${ }^{3-5}$ However, they are called mixed adenoneuroendocrine carcinoma when at least $30 \%$ of either component is present. $^{2}$

GEP-NECs are very rare. The incidence rate of colorectal NECs is 2:1,000,000, and is even lower for midgut/appendiceal sites. Pancreatic NECs account for $<1 \%$ of all pancreatic carcinomas, and $<2 \%-3 \%$ of all pancreatic NENs. ${ }^{6}$ Although somatostatin analogs, targeted therapies and peptide radio receptor therapy play an important role in the management of metastatic (m)GEP-NETs, chemotherapy is the main
Correspondence: Alberto Bongiovanni Osteoncology and Rare Tumors Center, Istituto Scientifico Romagnolo per lo Studio e la Cura dei Tumori (IRST) IRCCS, Via Piero Maroncelli, 40, 47014 Meldola, FC, Italy Tel +390543739100 Fax +39 0543739 I 23 Email alberto.bongiovanni@irst.emr.it 
therapeutic option for NECs and prognosis is poor, with a 2 -year survival $<20 \%$ and a median overall survival (mOS) of 5 months in a metastatic setting. ${ }^{7}$

GEP-NECs are usually treated in a similar way to smallcell lung cancer (SCLC), ie, etoposide associated with platinum compounds such as cisplatin or carboplatin. ${ }^{8}$ This drug combination is recommended by both European Neuroendocrine Tumor Society and North American Neuroendocrine Tumor Society guidelines. However, the few studies focusing on this regimen in SCLC did not evaluate it in GEP-NECs. In addition, biological affinity between SCLC and GEPNECs is questionable because the latter group also includes large-cell histology. Furthermore, although carboplatin is a potential alternative to cisplatin in terms of efficacy, specific studies on these two drugs in NECs are lacking. ${ }^{9-12}$

We retrospectively evaluated the efficacy of an etoposide and cisplatin or carboplatin regimen in metastatic GEP-NEC patients.

\section{Patients and methods}

Twenty patients (ten males and ten females) were treated at our institution with a platinum compound- and etoposide-based regimen between April 2010 and October 2014. The patients were required to have histologically confirmed, measurable metastatic large- or small-cell GEP-NECs according to the WHO 2010 classification, while those with mixed tumors were excluded. The patients with neutropenia $<1,500 \mathrm{~mm}^{3}$, thrombocytopenia $<100,000 \mathrm{~mm}^{3}$, or uncontrolled infection were also excluded. Clinical data were retrieved from the Rare Tumor Database and the project was reviewed and approved by the Medical Scientific Committee and Ethics Committee of Istituto Scientifico Romagnolo per lo Studio e la Cura dei Tumori (IRST) IRCCS. All patients had previously consented to treatment and had signed a consent form authorizing the processing of their personal data.

The staging procedures performed before starting treatment included physical examination, biochemical profile, and brain-chest-abdominal computerized tomography (CT) scan. Seventeen of the 20 patients underwent both gallium-68 $\left(\mathrm{Ga}^{68}\right)$ and fluorodeoxyglucose positron emission tomography (PET)/CT scans. The treatment was discontinued in the event of worsening clinical conditions, unacceptable toxicity, or disease progression. The patients were evaluated every three cycles or if disease progression was suspected, and those without radiological restaging were defined as non-evaluable.

In accordance with the international guidelines, none of the patients received somatostatin analogs. Second-line treatment was given to 12 patients. The response to treatment was evaluated using response evaluation criteria in solid tumor parameters. ${ }^{13}$ Toxicity was assessed using National Cancer Institute Common Terminology Criteria for Adverse Events version 4.0. Progression-free survival (PFS) was defined as the time from the start of treatment to disease progression or death. The OS was defined as the time from the start of treatment to death or a last known follow-up.

\section{Treatment}

All patients who received at least one cycle of cisplatin or carboplatin associated with etoposide were considered eligible for the study. Cisplatin was administered $25 \mathrm{mg} / \mathrm{m}^{2}$ on days 1-3 followed by $100 \mathrm{mg} / \mathrm{m}^{2}$ etoposide on days $1-3$ every 21 days. Carboplatin was administered $300 \mathrm{mg} / \mathrm{m}^{2}$ on day 1 followed by etoposide $100 \mathrm{mg} / \mathrm{m}^{2}$ on days $1-3$. Antiemetic prophylaxis using ondansetron and methylprednisolone was systematically proposed and was reinforced when necessary. All patients on cisplatin treatment were hydrated according to clinical practice guidelines. Chemotherapy was stopped in the event of unacceptable or life-threatening side-effects, performance status deterioration (ie, Eastern Cooperative Oncology group-performance status, 3), and/or tumor progression confirmed in imaging studies.

\section{Safety and efficacy}

Baseline assessment included medical history, physical examination with Eastern Cooperative Oncology groupperformance status evaluation, and biological tests (blood cell count, serum creatinine, and creatinine clearance according to Cockroft-Gault formula, bilirubin, alkaline phosphatase). During the treatment period, blood tests, toxicity evaluation, and physical examination were performed before each cycle. The tumor response rate was assessed by CT scan at 3-month intervals according to response evaluation criteria in solid tumor criteria. ${ }^{13}$ Evaluation procedures were performed ahead of schedule if a patient's general condition had deteriorated or when severe toxicity occurred.

\section{Statistical analysis}

Continuous variables were expressed as median and range, and categorical variables were expressed as frequency. PFS and OS were estimated with the Kaplan-Meier method (two-sided 95\% confidence intervals [CIs]) and the role of stratification factor was analyzed with the log-rank test. Estimated hazard ratios and their 95\% CI were calculated from a Cox regression model in univariate and multivariate analyses. Reported $P$-values $<0.05$ were used as threshold for significance. Statistical analyses were carried out with STATA/MP 10.1 for Windows (Stata Corp LP, College Station, TX, USA). 


\section{Results}

\section{General patient characteristics}

The clinical features of patients are shown in Table 1. The patients received a median number of six courses (range: 1-6)

Table I Patient characteristics

\begin{tabular}{|c|c|c|}
\hline Characteristics & $\mathbf{n}$ & $\%$ \\
\hline Median age, years (range) & $60(33-77)$ & \\
\hline \multicolumn{3}{|l|}{ Sex } \\
\hline Male & 10 & 50 \\
\hline Female & 10 & 50 \\
\hline \multicolumn{3}{|l|}{ ECOG } \\
\hline 0 & II & 55 \\
\hline 1 & 6 & 30 \\
\hline 2 & 3 & 15 \\
\hline \multicolumn{3}{|l|}{ Primary tumor site } \\
\hline Pancreas & 7 & 35 \\
\hline Stomach & 8 & 40 \\
\hline Colorectum & 5 & 25 \\
\hline \multicolumn{3}{|l|}{ Hisological subtype } \\
\hline Small-cell & 13 & 65 \\
\hline Large-cell & 7 & 35 \\
\hline \multicolumn{3}{|l|}{ BMI } \\
\hline$\leq 25$ & II & 58 \\
\hline$>25$ & 8 & 42 \\
\hline \multicolumn{3}{|l|}{ Number of metastatic sites } \\
\hline 1 & 2 & 10 \\
\hline$\geq 2$ & 18 & 90 \\
\hline \multicolumn{3}{|l|}{ Metastatic sites } \\
\hline Liver & 17 & 85 \\
\hline Lung & 6 & 30 \\
\hline Lymph nodes & 17 & 85 \\
\hline Bone & 4 & 20 \\
\hline Other & 9 & 45 \\
\hline \multicolumn{3}{|l|}{$\mathrm{Ga}^{68} \mathrm{PET} / \mathrm{CT}$} \\
\hline Positive & 7 & 35 \\
\hline Negative & 12 & 60 \\
\hline $\mathrm{NE}$ & 1 & 5 \\
\hline \multicolumn{3}{|l|}{ FDG PET/CT } \\
\hline Positive & 16 & 80 \\
\hline Negative & I & 5 \\
\hline NE & 3 & 15 \\
\hline \multicolumn{3}{|l|}{$\mathrm{Ga}^{68} / \mathrm{FDG}$ PET/CT } \\
\hline Both positive & 7 & 35 \\
\hline Both negative & I & 5 \\
\hline \multicolumn{3}{|l|}{ Treatment } \\
\hline Cisplatin/etoposide & 15 & 7 \\
\hline Carboplatin/etoposide & 5 & 2 \\
\hline \multicolumn{3}{|l|}{ Second line } \\
\hline Yes & 12 & 60 \\
\hline Chemotherapy & 10 & 50 \\
\hline PRRT & 2 & 10 \\
\hline No & 8 & 40 \\
\hline \multicolumn{3}{|l|}{ Ki67 (\%) } \\
\hline$\leq 55$ & 11 & 58 \\
\hline$>55$ & 8 & 42 \\
\hline
\end{tabular}

Abbreviations: BMI, body mass index; CT, computerized tomography; ECOG, Eastern Cooperative Oncology group-performance status; FDG, fluorodeoxyglucose $\mathrm{Ga}^{68}$, gallium-68; NE, not evaluable; PET, positron emission tomography; PRRT, peptide radio receptor therapy. of chemotherapy. A combination of cisplatin and etoposide was given to 15 patients while five received a carboplatin and etoposide-based regimen. The site of the primary tumor was pancreas (seven patients), stomach (eight), or colorectum (five). All patients had metastatic disease. The median Ki67 index was $45 \%$ (range: $25 \%-80 \%$ ). The treatment was stopped in one patient from each regimen group (10\%) due to worsening clinical conditions and in two $(10 \%)$ patients receiving the cisplatin combination because of progression.

\section{Safety evaluation}

Seventeen (85\%) patients had grade 3-4 hematological toxicity, of whom eight experienced at least one episode of grade 3 neutropenia and nine developed grade 4 neutropenia without fever. No deaths occurred due to toxicity. Non-hematological toxicities were observed in two patients, in particular nausea/vomiting, infection, and hypertransaminasemia, all G2 (Table 2).

\section{Tumor response rate}

Of the 20 patients treated, 19 were evaluable for tumor response. Of these, 13 (68\%) obtained a partial response, four (21\%) showed stable disease, and two (11\%) progressed.

\section{Survival}

Median follow-up was 19.6 months (range: 2.9-53.1). Median (m)OS was 13.5 months (95\% CI: 6.7-not reached [NR]), with an 18-month OS of 44.7\% (95\% CI: 19.9-67.0; Figure 1). Median PFS (mPFS) was 10.9 months (95\% CI: 6.2-19.3), with a 1-year PFS of 45.0\% (95\% CI: 20.4-67.1; Figure 2). No difference in survival was observed on the basis of sex or age (data not shown).

Patients with a positive $\mathrm{Ga}^{6}{ }^{6} \mathrm{PET} / \mathrm{CT}$ scan had a higher, albeit not significantly, OS than those with negative results ( $75 \%$ of positive patients were still alive at 18 months [95\% CI: $12.8-96.1$ ] vs $34.3 \%$ [95\% CI: 8.9-62.3] of those with negative $\mathrm{Ga}^{68} \mathrm{PET} / \mathrm{CT}$; $P$-value $=0.069$; Figure 3). $\mathrm{mPFS}$ was 19.3 months (95\% CI: 3.6-NR) and 6.3 months $(95 \%$ CI: $2.6-17.7 ; P$-value $=0.015)$ in GEP-NEC patients with

Table 2 Toxicity

\begin{tabular}{lllll}
\hline Toxicity & Grading & Male (\%) & Female (\%) & n (\%) \\
\hline Neutropenia & G3/G4 & 44 & 56 & I6 (80.0) \\
Neutropenia & G2 & 66 & 34 & $3(15.0)$ \\
Anemia & GI/G2 & 27 & 73 & II (55.0) \\
Nausea/vomiting & G2 & 100 & 0 & $2(10.0)$ \\
Hypertransaminasemia & G2 & 0 & 100 & I (5.0) \\
Thrombocytopenia & G3 & 100 & 0 & I (5.0) \\
Thrombocytopenia & GI & 0 & 100 & $3(15.0)$ \\
Infection & G2 & 100 & 0 & I (I0.0) \\
\hline
\end{tabular}




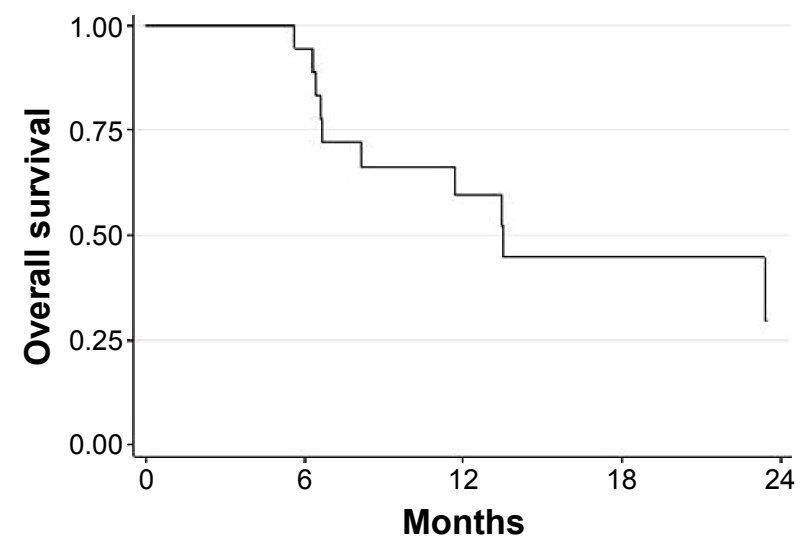

Figure I Median overall survival in the entire population.

Ki67 $<55 \%$ or $>55 \%$, respectively. mOS was $8.1(95 \%$ CI: 5.6-13.5) months in the latter group and was not reached in the Ki67 $<55 \%$ group ( $P$-value $=0.039$; Figure 4$)$. Patients with a lower body mass index (BMI) had a better prognosis in terms of both OS and PFS. mOS in this group was not reached, while the BMI $\geq 25$ group had a mOS of 11.7 months (95\% CI: 5.6-13.5; $P$-value 0.029; Figure 5). The former had a mPFS of 19.3 months (95\% CI: $2.6-\mathrm{NR})$, while a higher BMI had a PFS of 6.2 months $(95 \% \mathrm{CI}$ : 3.3-10.9; $P$-value $=0.006$ ).

Cox regression models were built including the variables Ki67 and BMI, both of which were significant in the log-rank test. Patients with Ki67 $>55 \%$ and BMI $\geq 25$ had a higher risk of death and disease progression. These variables did not have a significantly negative effect on death or progression in multivariate analysis (Table 3).

\section{Discussion}

GEP-NECs are very rare tumors with an incidence of $2: 1,000,000$ persons per year ${ }^{6}$ and represent only $5 \%-10 \%$ of all NEN. ${ }^{14}$ Given the similarity between NECs and SCLC,

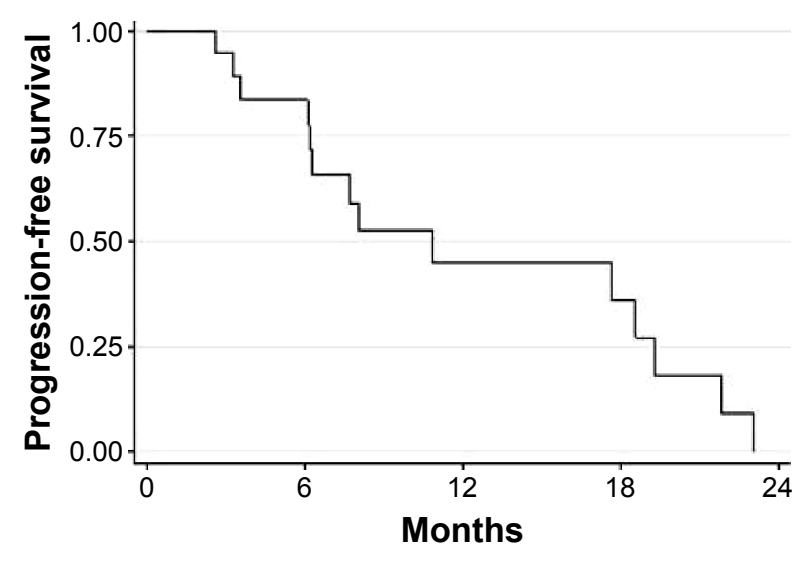

Figure 2 Median progression-free survival in the entire population.

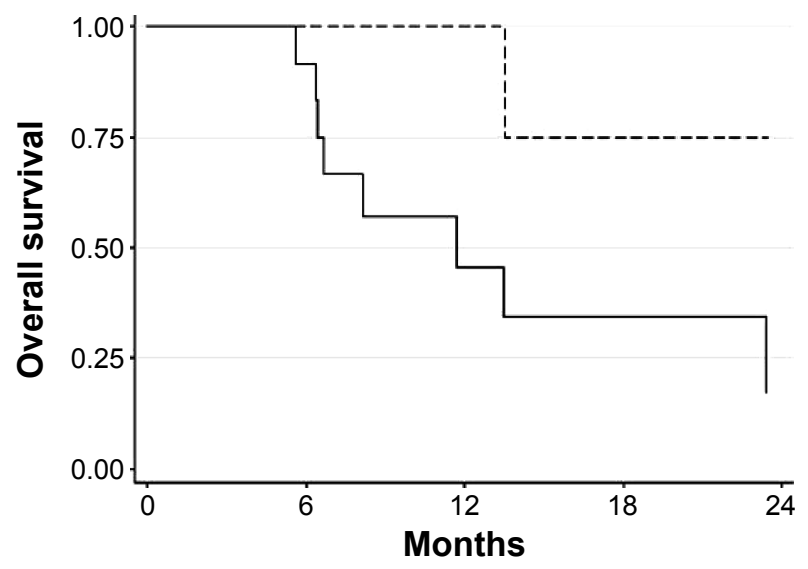

Ga ${ }^{68} \mathrm{PET} / \mathrm{CT}$ negative $----\cdot \mathrm{Ga}^{68} \mathrm{PET} / \mathrm{CT}$ positive

Figure 3 Median OS according to $\mathrm{Ga}^{68} \mathrm{PET} / \mathrm{CT}$ ( $P$-value $\left.=0.06\right)$.

Abbreviations: OS, overall survival; Ga ${ }^{68} \mathrm{PET} / \mathrm{CT}$, gallium- 68 positron emission tomography and computerized tomography.

the cisplatin-etoposide combination is the most widely used chemotherapy for NECs. However, unlike SCLC, studies using this regimen in GEP-NECs are lacking. Both tumor types show a number of molecular differences, eg, Bcl-2 overexpression in SCLC, or the retention of both short arms of chromosome 3 in extra-pulmonary NECs. ${ }^{15}$ In the latter, no information is available on the use of carboplatin as an alternative to cisplatin in terms of efficacy and toxicity. Furthermore, GEP-NECs include both small- and large-cell histologies, and there are no clinical features that can facilitate treatment decision making. A combination of cisplatin and etoposide was used by Moertel et $\mathrm{al}^{9}$ in 45 patients with NETs, 18 of whom had anaplastic NECs. Median time to progression and mOS of this subgroup were 11 and 19 months, respectively. In another retrospective analysis of patients with poorly or well-differentiated NETs treated with cisplatin and etoposide, mPFS was 9 months and mOS 15 months in the poorly differentiated group, some of whom received octreotide together with chemotherapy..$^{10}$ Both studies are somewhat dated and were conducted long before the WHO 2010 classification. At the time of their publication, no information was available on predictive factors of response to treatment.

In contrast, a retrospective analysis published in 2010 by Iwasa et $\mathrm{al}^{12}$ showed a low activity of the cisplatin-etoposide combination in NECs of the pancreato-biliary tract. In particular, 21 patients with hepato-biliary-pancreatic NECs were treated with first-line cisplatin and etoposide with an overall response rate of $14 \%$ and an mPFS and mOS of 1.8 and 5.8 months, respectively. In a recent paper by Lu et al, ${ }^{16}$ 16 patients with locally advanced or metastatic GEP-NECs 

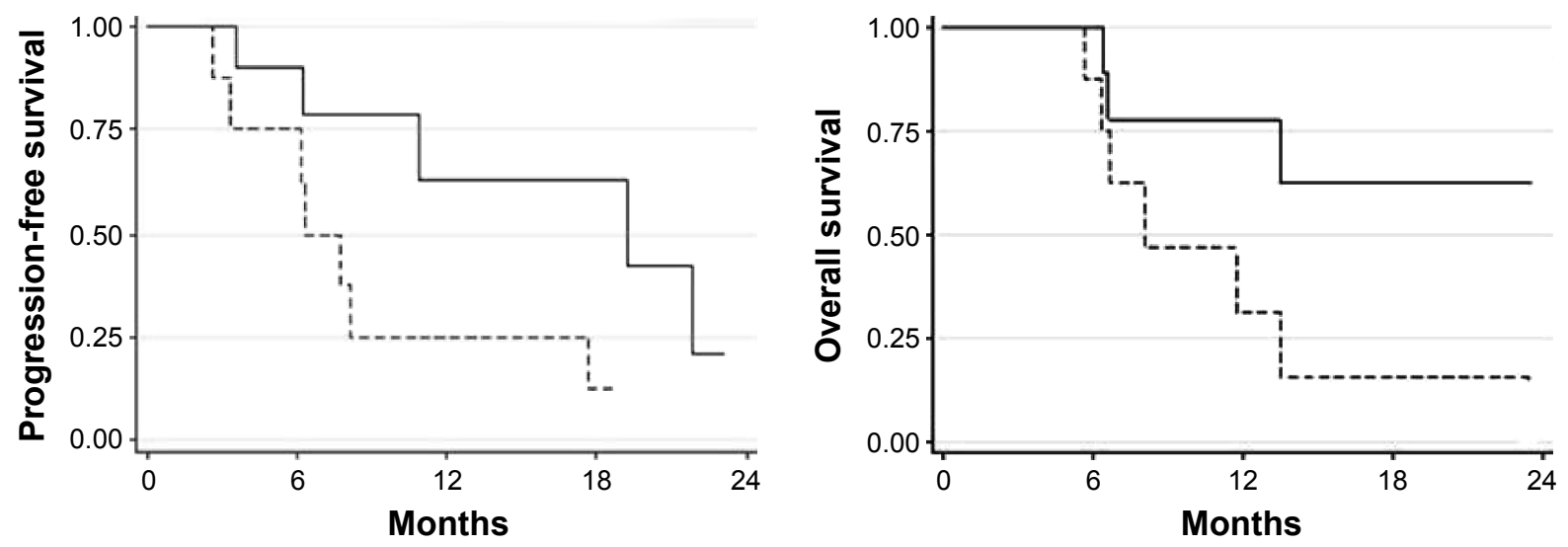

$-\mathrm{Ki} 67 \leq 55 \% \quad==-=-\mathrm{Ki} 67>55 \%$

Figure 4 Median PFS and OS by Ki67 ( $\leq 55 \%$ vs $>55 \%$ ).

Abbreviations: OS, overall survival; PFS, progression-free survival; vs, versus.

were treated with a combination of irinotecan and cisplatin. mPFS was 5.5 months and mOS was 10.6 months.

In our study, a platinum-containing regimen (cisplatin or carboplatin) associated with etoposide was used as a firstline treatment in metastatic GEP-NEC patients, obtaining a response rate of $68 \%$ and a mPFS of 13.5 months. No differences in efficacy were seen between the use of carboplatin or cisplatin.

The current WHO classification places all neuroendocrine GEP tumors with a Ki67 index $>20 \%$ in the "NEC" category, which clearly needs to be re-evaluated. A proposed amendment involves the separation of high-grade tumors according to morphology, creating a "well-differentiated high-grade NET" category and a "poorly differentiated high-grade NEC" category. ${ }^{17}$ Another proposal is to divide high-grade tumors into two proliferative categories, ie, those with Ki67 index $20 \%-55 \%$ and those with $\mathrm{Ki} 67$ index $>55 \%$. In our analysis, these two Ki67 categories had different outcomes, with

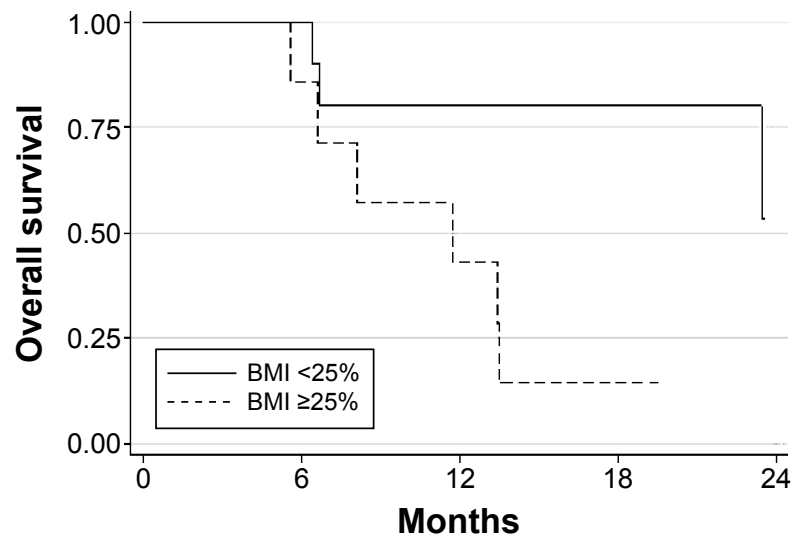

Figure 5 Median OS according to BMI $(P$-value $=0.029)$. Abbreviations: BMI, body mass index; OS, overall survival. a better mPFS and mOS in the lower $\mathrm{Ki} 67 \%$ group. Until a more accurate classification has been made for patients with NEC with a relatively low Ki67 index (20\%-55\%), factors such as morphology (poorly differentiated vs well differentiated), mitotic rate, and clinical course should also be taken into consideration before deciding upon treatment.

$\mathrm{Ga}^{68} \mathrm{PET} / \mathrm{CT}$ is a clinically useful imaging technique for the staging of NETs, while fluorodeoxyglucose PET has a prognostic role in this setting. ${ }^{18}$ In our study, patients with positive $\mathrm{Ga}^{68} \mathrm{PET} / \mathrm{CT}$ showed a better outcome than those with negative $\mathrm{Ga}^{68} \mathrm{PET} / \mathrm{CT}$, possibly due to higher differentiation. Both imaging studies add complementary information to the Ki67 value.

The relationship between BMI and chemotherapy outcome has mainly been studied in breast cancer. ${ }^{19,20}$ The relationship between adipose tissue and cancer, and adipose tissue and chemotherapy has also been investigated. The chemical composition of adipose tissue may positively affect the efficacy of chemotherapy. In fact, elevated fatty acid levels in adipose breast tissue have been shown to increase the degree of tumor shrinkage after primary chemotherapy in breast cancer patients. ${ }^{21}$ In our study, patients with a BMI $\geq 25 \mathrm{~kg} / \mathrm{m}^{2}$ had a poorer prognosis than those with a lower BMI. The effect of obesity-related factors on cell response to standard chemotherapy was recently studied in vitro in colorectal cancer cells. ${ }^{22}$ The authors showed that obesity-related conditions of elevated glucose, insulin, and insulin-like growth factor levels may increase cell viability and, in selected cases, resistance to chemotherapy and may also regulate hypoxia-inducible factor- $1 \alpha .^{22}$ The impact of such factors on NEN warrants investigation in both in vitro and in vivo settings. 
Table 3 Univariate and multivariate Cox regression models

\begin{tabular}{|c|c|c|c|c|}
\hline \multirow[t]{2}{*}{ Model } & \multicolumn{2}{|l|}{ OS } & \multicolumn{2}{|l|}{ PFS } \\
\hline & HR (95\% Cl) & $P$-value & HR (95\% Cl) & $P$-value \\
\hline \multicolumn{5}{|l|}{ Univariate } \\
\hline Only Ki67\% (>55 vs $\leq 55)$ & $3.81(0.98-14.87)$ & 0.054 & $4.58(1.20-17.43)$ & 0.026 \\
\hline Only BMI ( $\geq 25$ vs $<25)$ & $5.00(1.00-24.91)$ & 0.049 & $7.17(I .45-35.5 I)$ & 0.016 \\
\hline \multicolumn{5}{|l|}{ Multivariate } \\
\hline $\mathrm{Ki} 67 \%(>55$ vs $\leq 55)$ & $2.66(0.64-11.07)$ & 0.179 & $2.94(0.7 I-12.24)$ & 0.138 \\
\hline BMI $(\geq 25$ vs $<25)$ & $3.42(0.67-17.57)$ & 0.140 & $5.03(0.97-26.11)$ & 0.054 \\
\hline
\end{tabular}

Abbreviations: $\mathrm{BMI}$, body mass index; $\mathrm{Cl}$, confidence interval; $\mathrm{HR}$, hazard ratio; OS, overall survival; PFS, progression-free survival; vs, versus.

\section{Conclusion}

Platinum-based chemotherapy associated with etoposide represents an effective therapy for patients with metastatic GEP-NECs, especially those with positive $\mathrm{Ga}^{68} \mathrm{PET} / \mathrm{CT}$. Although our study is somewhat limited by its retrospective nature and by the small number of patients involved, it nevertheless provides valuable information on the therapeutic management of this rare group of tumors. Further prospective trials are needed to validate the therapeutic approach proposed and to define prognostic factors that could help to identify highly aggressive disease.

\section{Acknowledgment}

The authors thank Ursula Elbling for editorial assistance.

\section{Disclosure}

The authors report no conflicts of interest in this work.

\section{References}

1. Oberg K. Neuroendocrine tumors of the digestive tract: impact of new classifications and new agents on therapeutic approaches. Curr Opin Oncol. 2012;24(4):433-440.

2. Bosman FT, Carneiro F, Hruban RH, Theise, ND. WHO classification of tumours of the digestive system. CAB Direct. 2010;417.

3. Bernick PE, Klimstra DS, Shia J, et al. Neuroendocrine carcinomas of the colon and rectum. Dis Colon Rectum. 2004;47(2):163-169.

4. Brenner B, Shah MA, Gonen M, Klimstra DS, Shia J, Kelsen DP. Smallcell carcinoma of the gastrointestinal tract: a retrospective study of 64 cases. Br J Cancer. 2004;90(9):1720-1726.

5. Burke AB, Shekitka KM, Sobin LH. Sobin Small cell carcinomas of the large intestine. Am J Clin Pathol. 1991;95(3):315-321.

6. Ahlman H, Nilsson O, McNicol AM, et al. Poorly-differentiated endocrine carcinomas of midgut and hindgut origin. Neuroendocrinology. 2008;87(1):40-46.

7. Mitry E, Rougier P. The treatment of undifferentiated neuroendocrine tumors. Crit Rev Oncol Hematol. 2001;37(1):47-51.

8. Mavroudis D, Papadakis E, Veslemes M, et al. A multicenter randomized clinical trial comparing paclitaxel-cisplatin etoposide versus cisplatinetoposide as first-line treatment in patients with small-cell lung cancer. Ann Oncol. 2001;12(4):463-470.
9. Moertel CG, Kvols LK, O’Connell MJ, Rubin J. Treatment of neuroendocrine carcinomas with combined etoposide and cisplatin. Evidence of major therapeutic activity in the anaplastic variants of these neoplasms. Cancer. 1991;68(2):227-232.

10. Mitry E, Baudin E, Ducreux M, et al. Treatment of poorly differentiated neuroendocrine tumours with etoposide and cisplatin. Br J Cancer. 1999; 81(8):1351-1355.

11. Fjällskog ML, Granberg DP, Welin SL, et al. Treatment with cisplatin and etoposide in patients with neuroendocrine tumors. Cancer. 2001; 92(5):1101-1107.

12. Iwasa S, Morizane C, Okusaka T, et al. Cisplatin and etoposide as firstline chemotherapy for poorly differentiated neuroendocrine carcinoma of the hepatobiliary tract and pancreas. Jpn J Clin Oncol. 2010;40(4): 313-318.

13. Therasse P, Arbuck SG, Eisenhauer EA, et al. Newguidelines to evaluate the response to treatment in solid tumors. European Organization for Research and Treatment of Cancer, National Cancer Institute of the United States, National Cancer Institute of Canada. J Natl Cancer Inst. 2000;92(3):205-216.

14. Nilsson O, Van Cutsem E, Delle Fave G, et al. Poorly differentiated carcinomas of the foregut (gastric, duodenal and pancreatic). Neuroendocrinology. 2006;84(3):212-215.

15. Pizzi S, Azzoni C, Bassi D, Bottarelli L, Milione M, Bordi C. Genetic alterations in poorly differentiated endocrine carcinomas of the gastrointestinal tract. Cancer. 2003;98(6):1273-1282.

16. Lu ZH, Li J, Lu M, et al. Feasibility and efficacy of combined cisplatin plus irinotecan chemotherapy for gastroenteropancreatic neuroendocrine carcinomas. Med Oncol. 2013;30(3):664.

17. Sorbye H, Strosberg J, Baudin E, Klimstra DS, Yao JC. Gastroenteropancreatic high-grade neuroendocrine carcinoma. Cancer. 2014;120(18): 2814-2823.

18. Bahri H, Laurence L, Edeline J, et al. High prognostic value of $18 \mathrm{~F}$ FDG PET for metastatic gastroenteropancreatic neuroendocrine tumors: a long-term evaluation. J Nucl Med. 2014;55(11):1786-1790.

19. Inomata $M$, Hayashi $R$, Tokui $K$, et al. Outcome and prognostic factors in patients with small cell lung cancer who receive third-linechemotherapy. Tumori. 2014;100(5):507-511.

20. Cihan YB. Relationship of body mass index with prognosis in breast cancer patients treated with adjuvant radiotherapy and chemotherapy. Asian Pac J Cancer Prev. 2014;15(10):4233-4238.

21. Bougnoux $P$, Germain E, Chajès V, et al. Cytotoxic drugs efficacy correlates with adipose tissue docosahexaenoic acid level in locally advanced breast carcinoma. Br J Cancer. 1999;79(11-12):1765-1769.

22. Volkova E, Robinson BA, Willis J, Currie MJ, Dachs GU. Marginal effects of glucose, insulin and insulin-like growth factor on chemotherapy response in endothelial and colorectal cancer cells. Oncol Lett. 2014;7(2):311-320. 
OncoTargets and Therapy

\section{Publish your work in this journal}

OncoTargets and Therapy is an international, peer-reviewed, open access journal focusing on the pathological basis of all cancers, potential targets for therapy and treatment protocols employed to improve the management of cancer patients. The journal also focuses on the impact of management programs and new therapeutic agents and protocols on

patient perspectives such as quality of life, adherence and satisfaction The manuscript management system is completely online and includes a very quick and fair peer-review system, which is all easy to use. Visit http://www.dovepress.com/testimonials.php to read real quotes from published authors.

Submit your manuscript here: http://www.dovepress.com/oncotargets-and-therapy-journal 